\title{
Uniform physical theory of diffraction equivalent edge implementation in general computer codes
}

\section{Johansen, Peter Meincke}

Published in:

Antennas and Propagation Society International Symposium, 1996. AP-S. Digest

Link to article, DOI:

10.1109/APS.1996.549713

Publication date:

1996

Document Version

Publisher's PDF, also known as Version of record

Link back to DTU Orbit

Citation (APA):

Johansen, P. M. (1996). Uniform physical theory of diffraction equivalent edge currents for implementation in general computer codes. In Antennas and Propagation Society International Symposium, 1996. AP-S. Digest (Vol. 2, pp. 784-787). IEEE. https://doi.org/10.1109/APS.1996.549713

\section{General rights}

Copyright and moral rights for the publications made accessible in the public portal are retained by the authors and/or other copyright owners and it is a condition of accessing publications that users recognise and abide by the legal requirements associated with these rights.

- Users may download and print one copy of any publication from the public portal for the purpose of private study or research.

- You may not further distribute the material or use it for any profit-making activity or commercial gain

- You may freely distribute the URL identifying the publication in the public portal 


\title{
Uniform Physical Theory of Diffraction Equivalent Edge Currents for Implementation in General Computer Codes
}

\author{
Peter M. Johansen \\ Electromagnetics Institute \\ Technical University of Denmark, DK-2800 Lyngby, Denmark
}

\begin{abstract}
New uniform closed-form expressions for physical theory of diffraction equivalent edge currents are derived for truncated incremental wedge strips. In contrast to previously reported expressions, the new expressions are well-behaved for all directions of incidence and observation and take a finite value for zero strip length. Consequently, the new equivalent edge currents are, to the knowledge of the author, the first that are well-suited for implementation in general computer codes.
\end{abstract}

\section{Introduction}

General computer codes for calculation of the scattering from 3-dimensional perfect conductors illuminated by a plane wave often employ a physical theory of diffraction (PTD) [1] formulation. In this formulation the scattered field is expressed as the sum of the physical optics (PO) field and the fringe wave (FW) field where the latter takes into account the diffraction caused by edges. One way to calculate a high-frequency approximation to the FW field is to integrate Michaeli's PTD equivalent edge current's (EEC's) [2] along the edges of the structure. The EEC's are determined from an analytical integration of the FW current (the exact current minus the PO current) along incremental strips on the canonical wedge. Michaeli has derived EEC's for un-truncated incremental strips [2]. These un-truncated EEC's are not well-suited for general computer codes due to the presence of the nonremovable Ufimtsev singularity [2] and the discontinuities of the calculated FW field across the current layers associated with the un-truncated strips. These problems are eliminated by using truncated strips leading to truncated EEC's. The only reported truncated EEC's for a wedge with arbitrary angle [3] appear to have nonremovable singularities, which give rise to numerical problems and thus hamper their applicability in general computer codes [4]. In this work, new truncated EEC's are derived which do not have the singularity problems of the previously reported expressions.

\section{The Concept of Truncated EEC's}

The truncated EEC's are represented by the electric current $I_{T}$ and the magnetic current $M_{T}$. A high-frequency approximation to the electric FW field is calculated numerically from a line integration along the illuminated part $C$ of the edges of the structure as [3, eq. (1)]

$$
\vec{E}^{f w} \sim j k \int_{C}\left(Z I_{T} \hat{s} \times(\hat{s} \times \hat{z})+M_{T} \hat{s} \times \hat{z}\right) \frac{\exp (-j k s)}{4 \pi s} d C
$$

where the time factor $\exp (j \omega t)$ is suppressed, $k$ is the wave number, $Z$ is the 
intrinsic impedance of the ambient medium, and $\vec{s}=s \hat{s}=s(\hat{x} \sin \beta \cos \phi+$ $\hat{y} \sin \beta \sin \phi+\hat{z} \cos \beta$ ) is the vector to the far-field observation point from the point of integration (see Fig. 1). The truncated EEC's are determined by a sum of two contributions, one from each of the two adjoining faces $A$ and $B$ at each edge. The contribution from face $A, M_{T}^{A}$ and $I_{T}^{A}$, is calculated by analytically integrating the FW current on the canonical wedge along the truncated incremental strip with length $l^{A}$ shown in Fig. 1. However, this integration cannot be performed exactly in closed form and therefore an asymptotic technique is necessary. To this end the truncated EEC's are conveniently expressed as the difference between the un-truncated EEC's and the correction EEC's

$$
M_{T}^{A}=M_{U T}^{A}-M_{\text {cor }}^{A} \quad \text { and } \quad I_{T}^{A}=I_{U T}^{A}-I_{\text {cor }}^{A}
$$

where the correction EEC's are found from [3, eq. (3)-(7)]

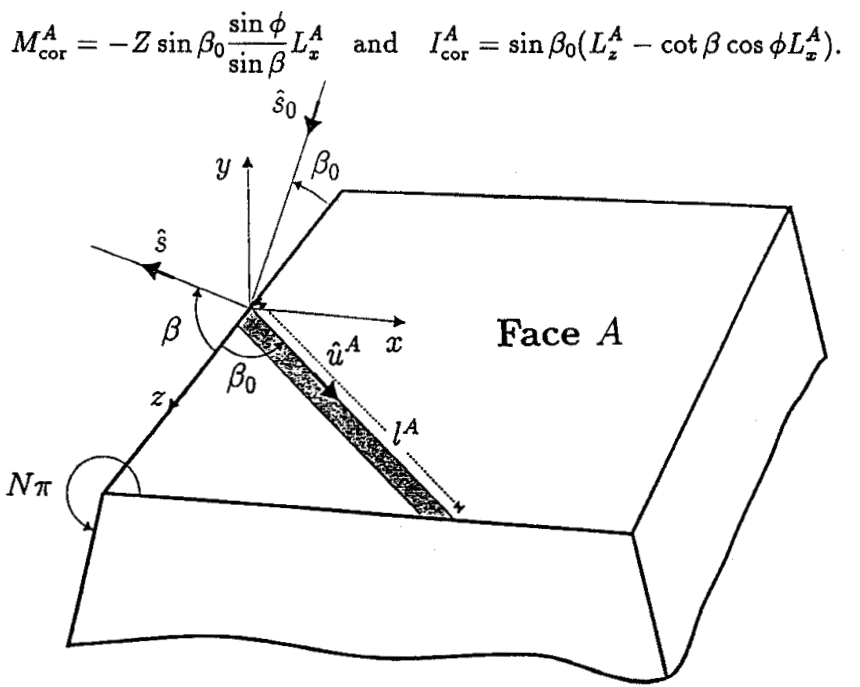

Figure 1: Flat face of a 3-dimensional structure.

In equation (3)

$$
L_{x, z}^{A}=\int_{l^{A}}^{\infty} J_{x, z}^{f w, A} \exp \left(j k u \hat{s} \cdot \hat{u}^{A}\right) d u
$$

where $J_{x, z}^{f w, A}$ denotes the $x$ - and $z$-components of the FW current on face $A$ and $\hat{u}^{A}=\hat{x} \sin \beta_{0}+\hat{z} \cos \beta_{0}$. The un-truncated EEC's can be expressed exactly in closed form [2, eq. (4)-(7)] whereas closed-form expressions for the correction EEC's can only be obtained from an asymptotic evaluation of the integral $L_{x, x}^{A}$ for $L=k l^{A} \sin ^{2} \beta_{0} \gg 1$ [3]. However, the asymptotic procedure applied in [3] gives rise to two problems in the correction EEC's when the exterior wedge angle $N \pi \neq 2 \pi[4]$. First, the correction EEC's tend to infinity as 
$L \rightarrow 0$. This can happen for edge points close to corners. Second, the correction EEC's contain nonremovable singularities which occur for various directions of incidence and observation.

New Truncated EEC's

In this work a new approach is used to perform the asymptotic calculation of the correction EEC's leading to new truncated EEC's using (2). The closed-form asymptotic expression for the FW current on a wedge, valid for $k x \sin \beta_{0} \gg 1$, is inserted into the integral $L_{x, z}^{A}$ in (4) and through a straightforward calculation of this integral the new result is obtained:

$$
\begin{aligned}
& M_{\text {cor }}^{A} \sim \frac{2 Z H_{z 0} \sin \phi \exp (j L(\mu-1))}{j k \sin \beta \sin \beta_{0}}\left[\frac{-\operatorname{sign}\left(\cos \frac{\phi_{0}}{2}\right)}{\mu+\cos \phi_{0}} F\left(\sqrt{2 L}\left|\cos \frac{\phi_{0}}{2}\right|\right)\right. \\
&+\left(\frac{\sqrt{1-\mu}}{\sqrt{2}\left(\mu+\cos \phi_{0}\right) \cos \frac{\phi_{0}}{2}}-\frac{\sqrt{2} \sin \frac{\pi}{N}}{N \sqrt{1-\mu}\left(\cos \frac{\pi}{N}-\cos \frac{\phi_{0}}{N}\right)}\right) F(\sqrt{L(1-\mu))}](5) \\
& I_{\text {cor }}^{A} \sim \frac{2 \exp (j L(\mu-1))}{j k \sin \beta_{0}\left(\mu+\cos \phi_{0}\right)}\left[\operatorname{sign}\left(\cos \frac{\phi_{0}}{2}\right)\right. \\
& \cdot\left(\frac{E_{z 0} \sin \phi_{0}}{Z \sin \beta_{0}}-H_{z 0}\left(\cot \beta_{0} \cos \phi_{0}+\cot \beta \cos \phi\right)\right) F\left(\sqrt{2 L}\left|\cos \frac{\phi_{0}}{2}\right|\right) \\
&+\sqrt{2(1-\mu)}\left(-\frac{E_{z 0} \sin \frac{\phi_{0}}{2}}{Z \sin \beta_{0}}+\frac{H_{z 0}}{2 \cos \frac{\phi_{0}}{2}}\left(\cot \beta_{0} \cos \phi_{0}+\cot \beta \cos \phi\right)\right. \\
&\left.\left.+\frac{H_{z 0} \sin \frac{\pi}{N}\left(\mu+\cos \phi_{0}\right)\left(\cot \beta_{0}-\cot \beta \cos \phi\right)}{N\left(\cos \frac{\pi}{N}-\cos \frac{\phi_{0}}{N}\right)(1-\mu)}\right) F(\sqrt{L(1-\mu)})\right](6)
\end{aligned}
$$

where $H_{z 0}$ and $E_{z 0}$ are the $z$-components of the incident magnetic and electric field, respectively, at the point of integration and $\phi_{0}+\pi$ is the azimuthal angle of the incident direction $\hat{s}_{0}$. Furthermore, $\mu=\left(\sin ^{2} \beta_{0}\right)^{-1}\left[\sin \beta_{0} \sin \beta \cos \phi+\right.$ $\left.\cos \beta_{0}\left(\cos \beta-\cos \beta_{0}\right)\right], \alpha$ is the solution to $\mu=\cos \alpha$ given in $[2$, eq. (8)], and $F$ is the modified Fresnel function given in [3, eq. (37)]. The above asymptotic expressions for $M_{\text {cor }}^{A}$ and $I_{\text {cor }}^{A}$ apply for $L=k l^{A} \sin ^{2} \beta_{0} \gg 1$. However, they are finite as $L \rightarrow 0$. The contribution from face $B$ is obtained by replacing $\beta_{0}$ with $\pi-\beta_{0}, \beta$ with $\pi-\beta, \phi_{0}$ with $N \pi-\phi_{0}, \phi$ with $N \pi-\phi$, and $l^{A}$ with $l^{B}$.

\section{Numerical Results}

Figure 2 shows a triangular 2-dimensional cylinder with side length $2 \lambda$ illuminated by a TM plane wave. The FW radar cross section is calculated using both the previously reported truncated EEC's, the new truncated EEC's and the method of moments applied to the electric field integral equation. Figure 3 shows that the nonremovable singularities in the previously reported expressions are not present when the new truncated EEC's are employed.

\section{References}

[1] P. Y. Ufimtsev, "Method of edge waves in the physical theory of diffraction," translation from russian prepared by the U.S. Air Force, Foreign Technology Division, Wright-Patterson AFB, OH, released for public distribution Sept. 7,1971 . 
[2] A. Michaeli, "Elimination of infinities in equivalent edge currents, part I: Fringe current components," IEEE Trans. Antennas Propagat., vol. 34, no. 7, pp. 912-918, July 1986.

[3] A. Michaeli, "Equivalent currents for second-order diffraction by the edges of perfectly conducting polygonal surfaces," IEEE Trans. Antennas Propagat., vol. 35, no. 2, pp. 183-190, Feb. 1987.

[4] P. M. Johansen and O. Breinbjerg, "A modified PO/PTD-technique for the analysis of radiation from a class of antennas mounted on conducting polyhedral structures," Progress In Electromagnetics Research Symposium, Noordwijk, The Netherlands, July 1994.

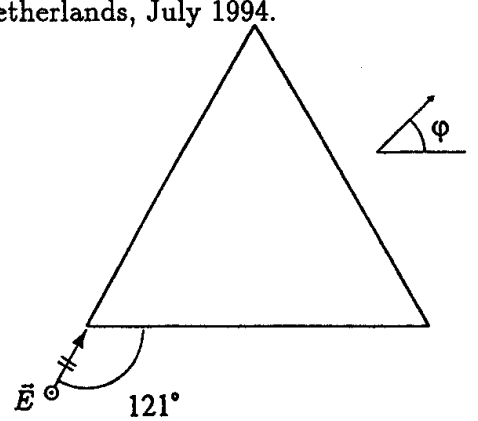

Figure 2: Cross-section of triangular cylinder illuminated by a TM plane wave.

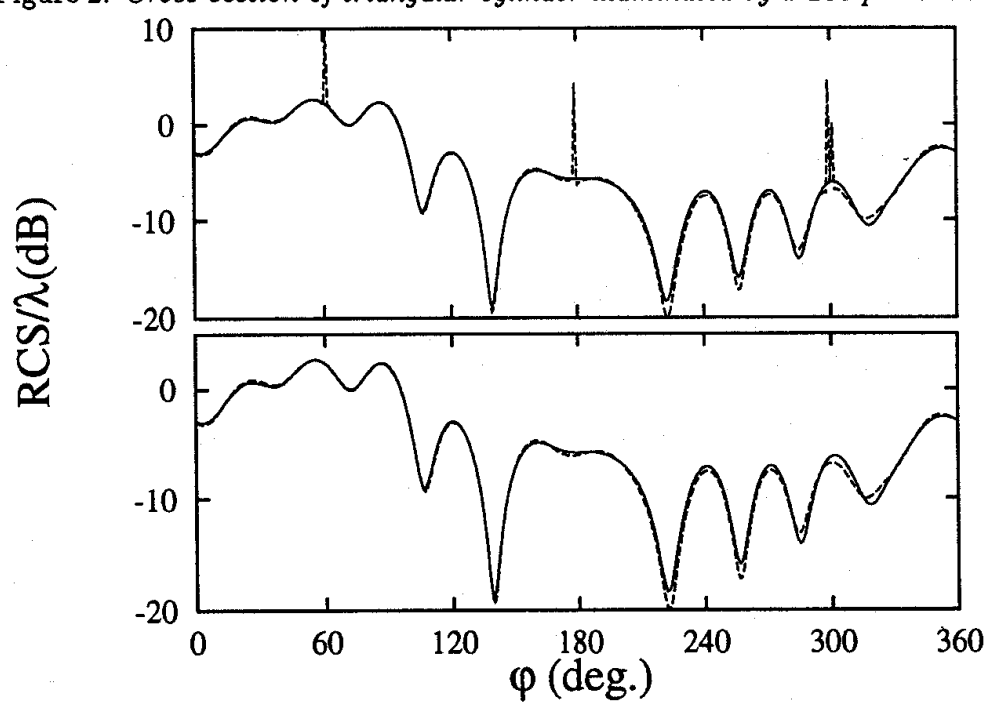

Figure 3: FW field for the configuration shown in Fig. 2. Solid line: EFIE minus PO. Dotted line in the top figure: Truncated EEC's reported in [3]. Dotted line in the bottom figure: New truncated EEC's. 